\title{
Impact of Interferences on Connectivity in Ad Hoc Networks
}

\author{
Olivier Dousse \\ School of Computer and \\ Communication Sciences \\ EPFL \\ CH-1015 Lausanne, Switzerland \\ Email: olivier.dousse@epfl.ch
}

\author{
François Baccelli \\ INRIA - ENS \\ Ecole Normale Supérieure \\ 45 , rue d'Ulm \\ F-75005 Paris, France \\ Email: francois.baccelli@ens.fr
}

\author{
Patrick Thiran \\ School of Computer and \\ Communication Sciences \\ EPFL \\ CH-1015 Lausanne, Switzerland \\ Email: patrick.thiran@epfl.ch
}

\begin{abstract}
We study the impact of interferences on the connectivity of large-scale ad-hoc networks, using percolation theory. We assume that a bi-directional connection can be set up between two nodes if the signal to noise ratio at the receiver is larger than some threshold. The noise is the sum of the contribution of interferences from all other nodes, weighted by a coefficient $\gamma$, and of a background noise.

We find that there is a critical value of $\gamma$ above which the network is made of disconnected clusters of nodes. We also prove that if $\gamma$ is non zero but small enough, there exist node spatial densities for which the network contains a large (theoretically infinite) cluster of nodes, enabling distant nodes to communicate in multiple hops. Since small values of $\gamma$ cannot be achieved without efficient CDMA codes, we investigate the use of a very simple TDMA scheme, where nodes can emit only every $n$-th time slot. We show qualitatively that it even achieves a better connectivity than the previous system with a parameter $\gamma / n$.
\end{abstract}

\section{INTRODUCTION}

Random graphs associated with the Poisson Boolean model and percolation properties of these graphs have been considered in [1] for analyzing the connectivity of ad hoc networks. Within this context, the Poisson Boolean model assumes that the stations are located according to a planar Poisson point process, and that each station has an independent random power, identically distributed for all stations.

A more physical model based on the signal to interference ratio was used within the context of ad hoc networks in [2]. In this last paper, which departs from a deterministic and finite population setting, all stations are assumed to have the same power, and some attenuation function is given. Station $A$ can receive a signal from station $B$ if the ratio of the power it receives from $B$ to the total power received from all other stations is above a threshold.

The same physical model was analyzed in [3] in the infinite plane case under Poisson assumptions within the context of CDMA networks. The corresponding coverage process has connection with Poisson shot noise processes.

The aim of the present paper is to bring all these approaches together and to study the connectivity of infinite ad hoc

The work presented in this paper was supported (in part) by the National Competence Center in Research on Mobile Information and Communication Systems (NCCR-MICS), a center supported by the Swiss National Science Foundation under grant number 5005-67322. networks under the physical model alluded to above. The parametric setting will be that of an homogeneous Poisson point process. Our main goal within this context is to learn whether the percolation phenomenon that was established in [1] for the case without interference still holds within this more realistic context.

By analogy with CDMA networks, we will introduce some orthogonality factor $\gamma$, which can vary from 0 to 1 , and which stems from the imperfect orthogonality of the codes used in CDMA. The case with $\gamma=0$ (perfect orthogonality) boils down to the case considered in [1].

As we will see, there are essential differences between the case $\gamma=0$ and $\gamma>0$. In some natural cases, for the same patterns, the first case could have an infinite component of the connectivity graph, whereas the second one could have no infinite component, or even no connectivity at all.

The main result of the paper is that under attenuation functions with finite support, percolation holds under conditions similar to those of the Boolean model of [1] provided the orthogonality factor $\gamma$ is small enough. In this sense, connectivity of ad hoc networks scales well with the size of the network even in the case of models that take interferences into account. The question whether this also holds true for attenuations of the type considered in practice (e.g. power functions with parameter between 3 and 6), over an infinite support, is still an open problem at this time.

The type of random graphs that are introduced in the paper are of independent interest. In particular, this class of random graphs which are built on the points of a Poisson point process, may simultaneously have infinite components, bounded range (each edge is of bounded length), and bounded degree (each vertex is of bounded degree).

As is it an essential feature, connectivity has received quite a lot of attention in the previous decade already, in the context of packet radio networks, and has gained renewed interest recently in the context of ad-hoc and sensor networks. Most results apply to the full connectivity of a network made of a finite number of nodes. A recursive formula giving the average number of hops between two connected nodes is found in [4], whereas the probability that a given number of nodes on a finite interval are all connected is computed in [5]. In the 2- 
dim. setting, relations between $k$-connectivity (the property that the graph has a minimal cutset equal to $k \geq 1$ ) and the node degree are studied in [6], whereas this problem is addressed when the transmission powers of the nodes are different in [7]. When the number of nodes $N$ tend to $\infty$, and when the distance $r$ below which nodes can connect decreases at a rate slower than $\sqrt{\log N / N}$, Gupta and Kumar have proven that all nodes are almost surely connected [8]. In this paper, we assume that the number $N$ of nodes is not fixed nor on a finite area, but that they are given as points of a Poisson process over the plane $\mathbb{R}^{2}$. We do not make assumptions on its intensity, so that our results also apply to low density areas. Since the number of nodes is not bounded, some of them will be disconnected. The problem is then related to percolation theory, which is to find the probability that a node belongs an infinite cluster of nodes. Since the pioneering work of Gilbert [9], which started the field of continuum percolation, the exact value of this probability is still an open problem. Some bounds on the critical intensity $\lambda^{*}$ below which it is zero have been obtained analytically in [9], [10], [11] for the Boolean Poisson Model, and numerically by many others [12]. Percolation of a clustered wireless network, in which the users (clients), who are distributed according to a Poisson process, are all covered by base stations that can connect to each other by a wireless link, is studied in [13]. This model reduces to the Poisson Boolean Model if one base station is placed at each client. To our knowledge, the percolation problem has not been addressed so far when interferences from other nodes are taken in account, which is the goal of this paper.

The paper is structured as follows. Section II describes the physical model considered for transmission between two nodes, from which the Poisson Signal To Interference Ratio Graph (STIRG) $\mathcal{G}(\gamma, \lambda)$ is derived. We obtain a bound on the node degree, which shows that is $\gamma$ is too large, the network is surely disconnected. We also show that for some attenuation functions, no connection is possible for any $\gamma>0$. It is important to know if a small but nonzero value of $\gamma$ still enables long range connectivity. In Section III, we prove that it is fortunately the case. We begin this section by some qualitative observations on simulations, and then formally prove our main result. Since percolation may hold for very small values of $\gamma>0$, narrow band communications may not be possible if we let all nodes emit simultaneously. A remedy is to use TDMA, so that each node is allowed to emit every $n$th time slot. One would expect that this solution is somewhat equivalent to dividing $\gamma$ by $n$. We find in Section IV that a very simple TDMA scheme does in fact achieve an even better connectivity that the previous one, with $\gamma / n$, and we provide a qualitative explanation for this property. Finally, we draw some conclusions and future perspective in Section V.

\section{MODEL}

We consider a multiple-hop ad-hoc network where nodes are distributed according to a Poisson point process of constant spatial intensity $\lambda$. Depending on its location, number of neighbors, and battery level, each node $i$ will adjust its emitting power $P_{i}$ within a given range $[0, P]$, where $P$ is the maximal power of a node, which is finite. The power of the signal emitted by Node $i$ and received by Node $j$ is $P_{i} L\left(\boldsymbol{x}_{i}-\boldsymbol{x}_{j}\right)$, where $\boldsymbol{x}_{i}$ and $\boldsymbol{x}_{j}$ are the positions of Node $i$ and $j$ in the plane, respectively, and $L(\boldsymbol{x})$ is the attenuation function in the wireless medium.

We assume that Node $i$ can transmit data to Node $j$ if the signal received by $j$ is strong enough, compared to the thermal noise. Formally, this condition is written as

$$
\frac{P_{i} L\left(\boldsymbol{x}_{i}-\boldsymbol{x}_{j}\right)}{N_{0}+\gamma \sum_{k \neq i, j} P_{k} L\left(\boldsymbol{x}_{k}-\boldsymbol{x}_{j}\right)} \geq \beta,
$$

where $N_{0}$ is the power of the thermal background noise and $\beta$ is the signal to noise ratio required for successful decoding. The coefficient $\gamma$ is the inverse of the processing gain of the system, it weights the effect of interferences, depending on the orthogonality between codes used during simultaneous transmissions. It is equal to 1 in a narrow band system, and is smaller than 1 in a broadband system that uses CDMA. The physical model of Gupta and Kumar [2] assumes $\gamma=1$; other models [14] allow $\gamma$ to be smaller than 1 .

Similarly, Node $j$ can transmit data to Node $i$ if and only if

$$
\frac{P_{j} L\left(\boldsymbol{x}_{j}-\boldsymbol{x}_{i}\right)}{N_{0}+\gamma \sum_{k \neq i, j} P_{k} L\left(\boldsymbol{x}_{k}-\boldsymbol{x}_{i}\right)} \geq \beta .
$$

From conditions (1) and (2), we can build an oriented graph that summarizes the available links between nodes. In order to define connected components, we have to introduce a symmetric relation. In this paper, we choose to neglect unidirectional links, which are difficult to exploit in wireless networks [15]. In other words, we declare that Node $i$ and Node $j$ are directly connected if and only if both (1) and (2) are satisfied. This new relation leads to the definition of a non-oriented random graph associated with the Poisson point process. This Poisson Signal To Interference Ratio Graph (STIRG) $\mathcal{G}(\gamma, \lambda)$ is the main object of study in the present paper.

\section{A. A Bound on the Degree of the Nodes}

In the following theorem, we will prove that if $\gamma>0$, the number of neighbors of each node is bounded from above (note that this is not the case in the Boolean Model with $\gamma=$ $0)$. bors.

Theorem 1: Each node can have at most $1+1 / \gamma \beta$ neigh-

Proof: Pick any node (called hereafter Node 0), and let $N$ be the number of its neighbors (i.e. the number of nodes to which Node 0 is connected). If $N \leq 1$, the claim is trivially proven. Suppose next that $N>1$, and denote by 1 the node whose signal power received by Node 0 is the smallest but is non zero, namely is such that

$$
P_{1} L\left(\boldsymbol{x}_{1}-\boldsymbol{x}_{0}\right) \leq P_{i} L\left(\boldsymbol{x}_{i}-\boldsymbol{x}_{0}\right), i=2 \ldots N .
$$

Since it is connected to Node 0, (1) imposes that

$$
\frac{P_{1} L\left(\boldsymbol{x}_{1}-\boldsymbol{x}_{0}\right)}{N_{0}+\gamma \sum_{i=2}^{\infty} P_{i} L\left(\boldsymbol{x}_{i}-\boldsymbol{x}_{0}\right)} \geq \beta .
$$


Taking (3) into account, (4) implies that

$$
\begin{aligned}
P_{1} L\left(\boldsymbol{x}_{1}-\boldsymbol{x}_{0}\right) \geq & \beta N_{0}+\beta \gamma \sum_{i=2}^{\infty} P_{i} L\left(\boldsymbol{x}_{i}-\boldsymbol{x}_{0}\right) \\
\geq & \beta N_{0}+\beta \gamma(N-1) P_{1} L\left(\boldsymbol{x}_{1}-\boldsymbol{x}_{0}\right) \\
& +\beta \gamma \sum_{i=N+1}^{\infty} P_{i} L\left(\boldsymbol{x}_{i}-\boldsymbol{x}_{0}\right) \\
\geq & \beta \gamma(N-1) P_{1} L\left(\boldsymbol{x}_{1}-\boldsymbol{x}_{0}\right),
\end{aligned}
$$

from which we deduce that

$$
N \leq 1+\frac{1}{\beta \gamma}
$$

In CDMA cellular networks, this kind of bound is known under the name of pole capacity (see e.g. [16], [3]).

As a consequence of Theorem 1 , we see that if $\gamma>1 / \beta$, each node has at most one neighbor. This is a very general and restrictive condition, that imposes the network to use efficient spread-spectrum encoding in order to keep $\gamma$ small, or to introduce a scheduling between nodes to avoid having them emitting all the same time - we will investigate such a scheme in Section IV.

\section{B. Shot-Noise}

The sum in the denominator of (1) is a random variable that depends on the position of almost all nodes in the network. We can write it as $N_{0}+\gamma I\left(\boldsymbol{x}_{j}\right)-\gamma P_{i} L\left(\boldsymbol{x}_{i}-\boldsymbol{x}_{j}\right)$ where

$$
I(\boldsymbol{x})=\sum_{i, \boldsymbol{x}_{i} \neq \boldsymbol{x}} P_{i} L\left(\boldsymbol{x}_{i}-\boldsymbol{x}\right)
$$

is the interference contribution. This kind of variable is called a Poisson shot-noise. As it is an infinite sum, it may diverge to infinity, making connections impossible.

If we assume that the sequence $\left\{P_{i}\right\}$ is i.i.d. and independent of the point process, and that $L(\boldsymbol{x})$ has the form $L(\boldsymbol{x})=l(\|\boldsymbol{x}\|)$ where $l(t)$ is a non increasing function of $t$, the necessary and sufficient condition for the sum

$$
\sum_{i} P_{i} L\left(\boldsymbol{x}_{i}-\boldsymbol{x}\right)
$$

to be a.s. finite is given in [17]:

$$
\int_{y}^{\infty} l(t) t d t<\infty, \text { for a sufficiently large } y \text {. }
$$

We notice that for $l(t)=1 / t^{2}$, the integral in (6) is divergent and thus no connection is possible in this case whenever $\gamma>0$.

By letting $y=0$ in (6), we obtain the condition for integrability, which is stronger. This last property holds for all stationary point processes with finite intensity see e.g. [18], and in particular in the homogeneous Poisson case.

\section{Attenuation}

For the attenuation, the most common function is

$$
l(t)=\frac{1}{t^{\alpha}}
$$

with $\alpha$ ranging from 3 to 6 . It makes sense to assume attenuation to be a bounded function in the vicinity of the antenna. The following two functions:

[A1] $l(t)=A \max \left(t, r_{0}\right)^{-\alpha}$,

[A2] $l(t)=(1+A t)^{-\alpha}$,

with $A>0$, are bounded modifications of the latter considered in [3].

\section{PERCOLATION}

The percolation probability is the probability that an arbitrary node belongs to a cluster of infinite size. The main result of percolation theory is that there exists a finite, positive value $\lambda^{*}$ of $\lambda$, under which the percolation probability is zero (subcritical phase) and above which it is non zero (super-critical phase). In the sub-critical phase, long range connections in multiple hops are not possible, contrary to the super-critical phase. It is thus a crucial property to establish in a network. We begin this central section by the much simpler Boolean model, which is a particular case for our model when $\gamma=0$. We then make some preliminary observations on simulations to show the difference between the graphs obtained when the interferences are neglected (which amounts to set $\gamma=0$ ) or not (when $\gamma>0$ ). We finally prove that percolation occurs for small, but nonzero values of $\gamma$.

\section{A. Existence of a percolation threshold for $\gamma=0$}

Let us first note that if we let $\gamma=0$, the model described in Section II becomes equivalent to a generalized Boolean model, where two nodes are connected if and only if they are in a ball of radius $r$ (which can be a deterministic or random value), independently from all the other nodes. Assuming all nodes emit at the maximum power $P$, this radius $r$ is then constant and found from (1) to be equal to

$$
r=\sup \left\{\rho \text { such that } l(\rho) \geq \frac{\beta N_{0}}{P}\right\} \text {. }
$$

For example, for the attenuation function (7), this radius reads $r=\left(P /\left(\beta N_{0}\right)\right)^{1 / \alpha}$. This is the model we have studied in [1], and for which many results from continuous percolation theory apply [10]. The most important one is mentioned above, namely that there is a critical density $\lambda^{*}$, above which the graph contains an infinite connected component.

\section{B. Some observations on the graph with $\gamma>0$}

If $\gamma<\gamma^{\prime}$, it is clear that for the same realization of the spatial point process giving the position of the nodes, the graph obtained with $\gamma^{\prime}$ misses some edges in the graph obtained with $\gamma$. In other words, $\mathcal{G}\left(\gamma^{\prime}, \lambda\right) \subseteq \mathcal{G}(\gamma, \lambda)$. As a result, it is not sure that percolation still occurs for nonzero values of $\gamma$. For any value of $\gamma \geq 0$, there is a density $\lambda_{1}(\gamma)$ such that for $\lambda<\lambda_{1}$ there is no percolation. Define

$\lambda^{*}(\gamma)=\sup \left\{\lambda^{\prime}\right.$ such that $\forall \lambda<\lambda^{\prime}$ there is no percolation $\}$. 


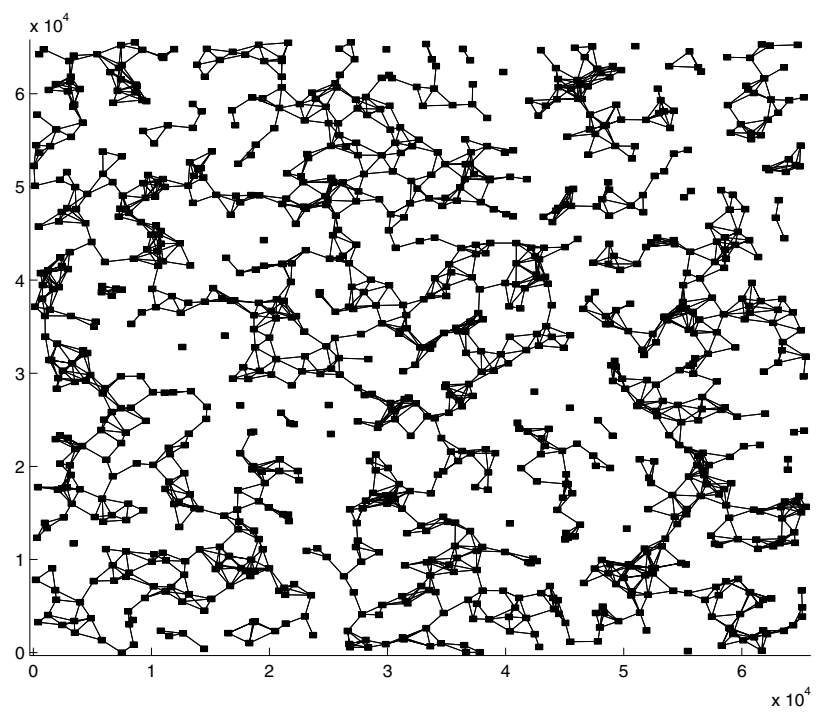

Fig. 1. An example of graph $\mathcal{G}(0, \lambda)$ with no interference (Boolean Model). As the node density is super-critical $\left(\lambda>\lambda^{*}(0)\right)$, most of the nodes belong to the same connected component. [This simulation was run in a square of $65,536 \times 65,536$ pixels with a node density such that the expected number of nodes in the square is 1,000 . The other parameters are $\alpha=3, \beta=1$, $\gamma=0, P_{i}=100,000 \forall i$ and $N_{0}=6 \times 10^{-6}$.]

If $\lambda^{*}(\gamma)$ is finite for at least some positive value of $\gamma$, it means that for some densities $\lambda \geq \lambda^{*}(\gamma)$, the graph $\mathcal{G}(\gamma, \lambda)$ contains an infinite connected component. Compared to the Boolean model, the critical intensity is thus now a function of $\gamma$, which must be increasing because of Lemma 1 below.

Lemma 1: Let $\gamma<\gamma^{\prime}$ be two positive real numbers. If both $\lambda^{*}(\gamma)$ and $\lambda^{*}\left(\gamma^{\prime}\right)$ are finite, then

$$
\lambda^{*}(\gamma) \leq \lambda^{*}\left(\gamma^{\prime}\right) .
$$

Proof: By definition of $\lambda^{*}\left(\gamma^{\prime}\right)$, one can find $\lambda \geq \lambda^{*}\left(\gamma^{\prime}\right)$ such that the graph $\mathcal{G}\left(\gamma^{\prime}, \lambda\right)$ contains an infinite connected component. Since $\mathcal{G}\left(\gamma^{\prime}, \lambda\right) \subseteq \mathcal{G}(\gamma, \lambda)$, we have that $\mathcal{G}(\gamma, \lambda)$ also contains an infinite connected component, and hence that $\lambda^{*}(\gamma) \leq \lambda$.

As $\lambda$ can be chosen arbitrarily close to $\lambda^{*}\left(\gamma^{\prime}\right)$, we have finally that $\lambda^{*}(\gamma) \leq \lambda^{*}\left(\gamma^{\prime}\right)$.

Up to now however, we only know that $\lambda^{*}(0)$ exists, and is the usual percolation threshold for a Boolean Model. The next subsection will prove that $\lambda^{*}(\gamma)$ is finite for finite, nonzero values of $\gamma$. In fact, instead of having a single value $\lambda^{*}(0)$ marking the transition between the sub-critical and supercritical phases, we will have a curve $\lambda^{*}(\gamma)$, as we now have two free parameters $\left(\gamma\right.$ and $\left.N_{0}\right)$ instead of only one $\left(N_{0}\right)$. The SNR threshold $\beta$ is kept as a parameter normalized to 1 in this paper. A different value simply amounts to scale the values of $\gamma$ and $N_{0}$ accordingly, by dividing them by the new value of $\beta$.

We have computed by simulation the value of the percolation threshold $\lambda^{*}(\gamma)$, with $L(\boldsymbol{x})=\|\boldsymbol{x}\|^{-\alpha}$. The simulation results are shown in Figures 1, 2, 3 and 4. In the simulations, all nodes emit with the same power $P$. We observe in Figure 1 and 2 that $\mathcal{G}(0.01, \lambda) \subseteq \mathcal{G}(0, \lambda)$. We also observe in Figure 3

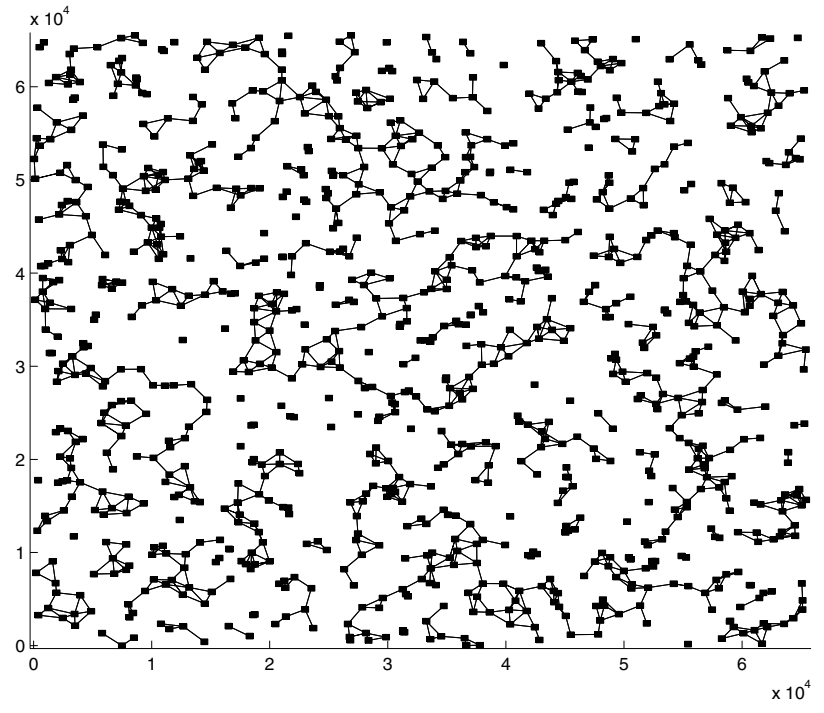

Fig. 2. An example of graph $\mathcal{G}(\gamma, \lambda)$ with interferences $(\gamma=0.01)$. This simulation was run with the same parameters as in Figure 1, except $\gamma$ that is now nonzero. Due to the interferences, the graph is split into many small components.

that percolation still occurs for sufficiently small values of $\gamma$, but disappears when $\gamma$ becomes too large. Actually, $\lambda^{*}(\gamma)$ diverges at some point $\gamma^{*}$. For $\gamma>\gamma^{*}$, percolation appears impossible, no matter how large the node spatial density is.

We know already from Theorem 1 , that if $\gamma>1 / \beta$, each node has at most one neighbor. In such a case, each component has at most two elements, making percolation impossible. This proves that $\gamma^{*}$ is less than $1 / \beta$.

Figure 4 illustrates the percolation phenomenon with $\gamma \cong$ $\gamma^{*}$, for the same spatial node distribution as in Figure 1 . Note how the interferences strongly affect the overall graph structure.

We also computed statistics on the number of neighbors of the nodes at the percolation threshold. The result is presented in Figure 5. We observe that fewer edges are needed to make the graph percolate when $\gamma>0$, and that the variance of the number of neighbors decreases rapidly when $\gamma$ increases.

\section{Percolation for nonzero values of $\gamma$}

We have shown above that if $\gamma$ exceeds some finite, positive critical value, percolation does not occur. We want now to show that percolation can occur for nonzero values of $\gamma$. We make the simplifying assumption that every node emits at maximal power $P: P_{i}=P \forall i$. This corresponds to the worst power assignment for the interfering communications. We need additional assumptions on the attenuation function $L(\boldsymbol{x})$. In this subsection, we restrict ourselves to non increasing, isotropic attenuation functions that have the following additional properties:

$$
\begin{array}{r}
L(\boldsymbol{x})=0 \forall \boldsymbol{x} \in \mathbb{R}^{2} \text { s.t. }\|\boldsymbol{x}\|>d \\
\frac{\beta N_{0}}{P}<L(\boldsymbol{x})<M \forall \boldsymbol{x} \in \mathbb{R}^{2} \text { s.t. }\|\boldsymbol{x}\| \leq \delta
\end{array}
$$




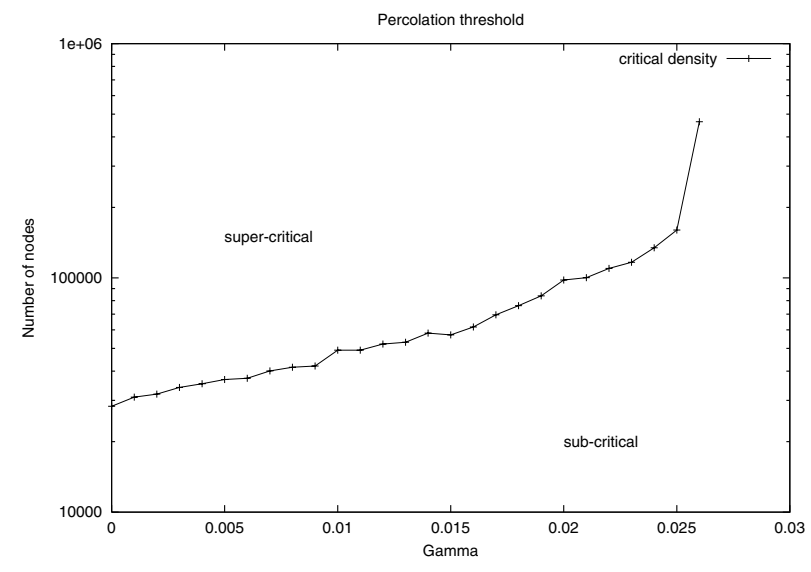

Fig. 3. Critical node density $\lambda$ as a function of $\gamma$. The curve shows the critical value of $\lambda$ above which the network percolates. One can observe that this value diverges when $\gamma$ increases. Actually, percolation only occurs for values of $\gamma$ smaller than a certain limit $\gamma^{*}$. Above this limit, no infinite connected component is observed, even with arbitrarily high node density. [The parameters of this simulation are $\alpha=3, \beta=1, N_{0}=10^{-3}$ and $P_{i}=100,000 \forall i$.]

for some $0<\delta<d$ and $M>\beta N_{0} / P$.

We will then prove the following main theorem.

Theorem 2: If the isotropic attenuation function $L(\boldsymbol{x})$ verifies assumptions (8) and (9), then there exists $\lambda_{c}<\infty$ such that for all $\lambda>\lambda_{c}$ there exists $0<\gamma_{c}(\lambda)$ such that for $\gamma<\gamma_{c}(\lambda)$ the probability that a node belongs to an infinite cluster is strictly greater than zero.

This theorem implies therefore that $\gamma^{*}>0$, and therefore that communication between distant nodes is still possible despite interferences. The proof of this central theorem is quite lengthy, and is therefore divided in several intermediate results. The first step is to map the process defined on the continuous plane $\mathbb{R}^{2}$ onto a discrete grid (lattice) $\mathcal{L}$, whose edges are declared open if certain properties of the Poisson process in their neighborhood are met. The second and more lengthy step is to prove bond percolation, that is, the existence of an infinite path made of open edges, on the dual lattice $\mathcal{L}^{\prime}$. The third step is then straightforward, as the reverse mapping allows us then to conclude that the network indeed percolates and has an infinite cluster on the continuous plane $\mathbb{R}^{2}$. The reason for carrying most of the proof on the discrete lattice $\mathcal{L}^{\prime}$ is that we can then make use of the larger collection of results found in the literature [19] on discrete bond percolation than on continuous percolation.

\section{Step 1: mapping of the graph on a lattice}

We begin by constructing a square lattice, denoted by $\mathcal{L}$ over the plane, with edge length $d$. Let $\mathcal{L}^{\prime}$ be the dual lattice of $\mathcal{L}$, obtained by putting a vertex in the center of every square of $\mathcal{L}$, and an edge across every edge of $\mathcal{L}$. As $\mathcal{L}$ is square lattice, $\mathcal{L}^{\prime}$ is simply the same lattice shifted by $d / 2$ horizontally and vertically, as depicted in Figure 6. Note that there exists a oneto-one relation between the edges of $\mathcal{L}^{\prime}$ and the edges of $\mathcal{L}$. Furthermore, we set the origin $O$ of the plane at a vertex of

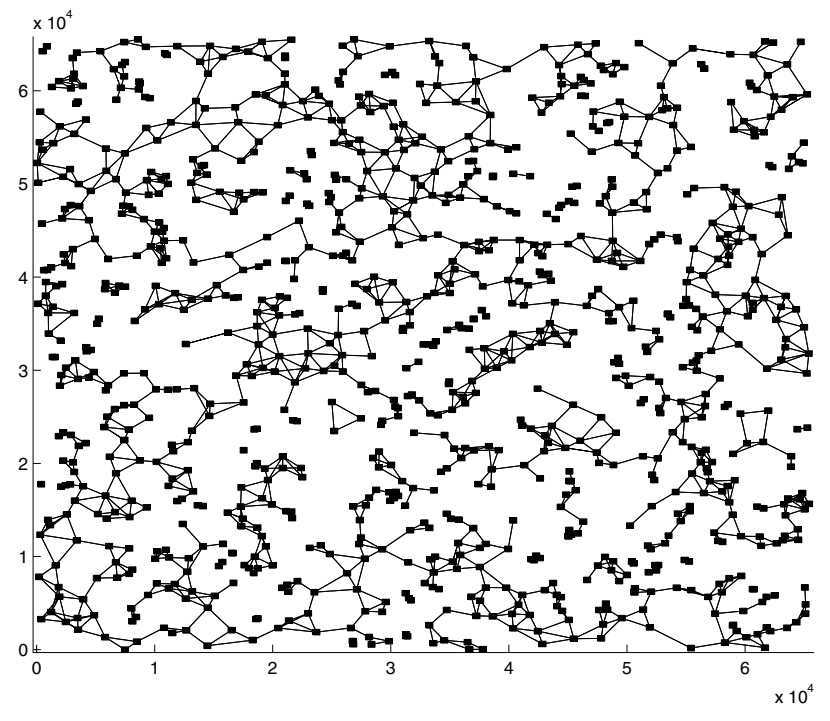

Fig. 4. A barely supercritical graph with interferences. This simulation was run with the same parameters as in Figure 1, except $\gamma=0.02$ and $N_{0}=$ $10^{-6}$. The graph percolates despite the interferences because the background noise $N_{0}$ is much weaker. One can observe that fewer edges are needed to achieve percolation than in Figure 1.

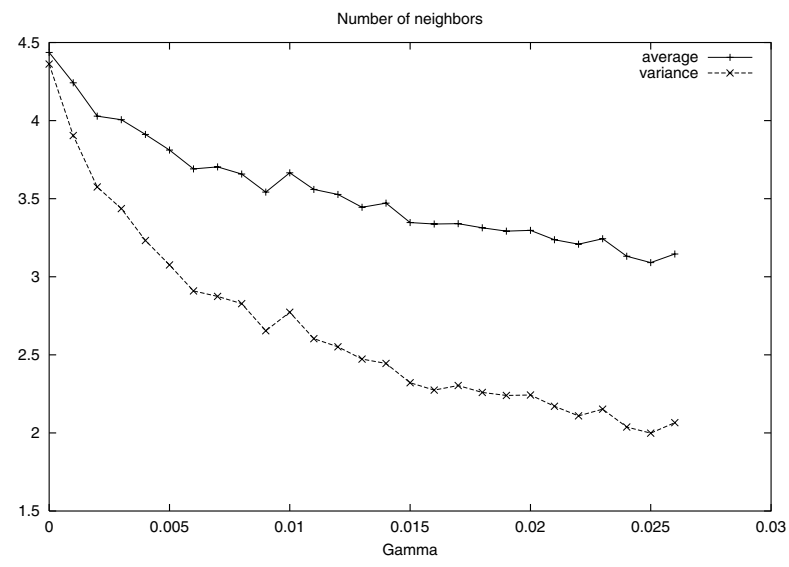

Fig. 5. Average number of neighbors and its variance as a function of $\gamma$. The average number of neighbors at percolation threshold decreases when $\gamma$ increases, meaning that fewer edges are needed for percolation in the model with interferences. For $\gamma=0$, the expected value and variance are equal because the number of neighbors is a Poisson random variable. [The parameters of this simulation are the same as in Figure 3]

$\mathcal{L}^{\prime}$, without any loss of generality.

Let us now consider the original Poisson point process over the plane. Each square of Lattice $\mathcal{L}$ contains in average $\lambda d^{2}$ points. We will study bond percolation on Lattice $\mathcal{L}^{\prime}$. To do this, we will declare some edges open and others closed depending on the realization of the underlying Poisson point process.

In Lattice $\mathcal{L}$, we divide again each square into $K^{2}$ subsquares of size $\frac{d}{K} \times \frac{d}{K}$, where $K \in \mathbb{N}^{*}$ is given by

$$
K=\lceil\sqrt{5} d / \delta\rceil .
$$




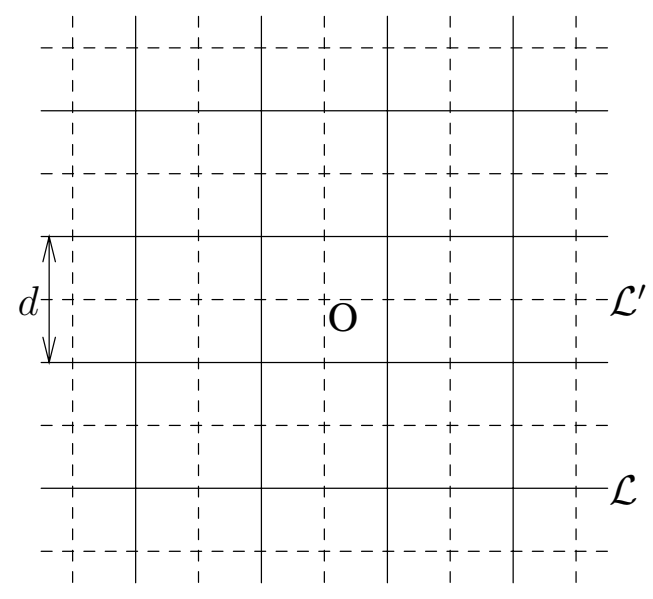

Fig. 6. Lattice $\mathcal{L}$ (plain) and its dual $\mathcal{L}^{\prime}$ (dashed)

This value has been chosen so that $\|\boldsymbol{x}\| \leq \sqrt{5} d / K$ implies that $\|\boldsymbol{x}\| \leq \delta$. Next, we introduce a second integer parameter $N$ defined by

$$
N=\inf _{\boldsymbol{x} \text { s.t. }\|\boldsymbol{x}\| \leq \sqrt{5} d / K}\left\lfloor\frac{1}{\gamma M}\left(\frac{P L(\boldsymbol{x})}{\beta}-N_{0}\right)\right\rfloor .
$$

Because of (9) and (10), one can check that $N \in \mathbb{N}$. Combining (9) with (10) and (11), we obtain the following inequality

$$
\|\boldsymbol{x}\| \leq \frac{\sqrt{5} d}{K} \Rightarrow \frac{L(\boldsymbol{x}) P}{N_{0}+\gamma N M} \geq \beta,
$$

which is more restrictive than the left inequality in (9).

We can now formally define our discrete percolation model from the original, continuous one by introducing a bunch of definitions. We designate by the term "point" the location of a node in the original network, to avoid any confusion with a vertex in the grid $\mathcal{L}$.

Definition 1: A square $X$ of $\mathcal{L}$ is said to be populated if all its subsquares contain at least one point.

Definition 2: An edge $\boldsymbol{a}$ of $\mathcal{L}$ is said to be open if the following conditions are fulfilled:

- both squares adjacent to $\boldsymbol{a}$ are populated, and

- both squares adjacent to $\boldsymbol{a}$ and all their direct neighbors (that is, all the squares having at least one vertex in common with these two squares, as depicted in Figure 7) do not contain more than $N$ points.

Definition 3: An edge $\boldsymbol{a}^{\prime}$ of $\mathcal{L}^{\prime}$ is said to be open if and only if the corresponding edge of $\mathcal{L}$ is open.

Definition 4: A path (in $\mathcal{L}$ or $\mathcal{L}^{\prime}$ ) is said to be open (resp. closed) if all edges forming this path are open (resp. closed).

The above definitions have been chosen such that an open edge guarantees connectivity in the continuous model (see Lemma 4 hereafter). In fact, the first condition ensures a homogeneous population in the squares, whereas the second condition puts a limit to the interference contribution. It is very important to notice that the main difference between this model and the usual discrete percolation models is that here

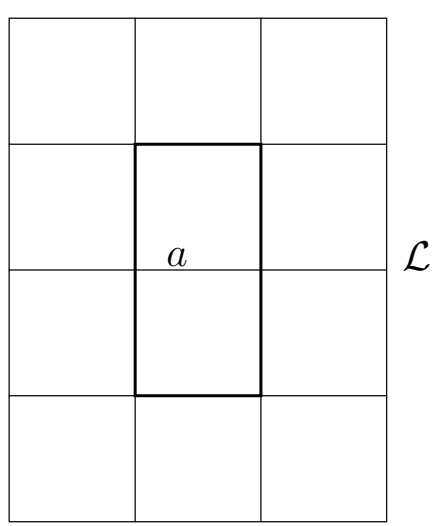

Fig. 7. Conditions for $a$ to be open: both squares in the middle (bold line) must be populated, and the total number of points in the 12 squares must be at most $N$

the state of the edges (open or closed) are not independent from each other.

\section{Step 2: percolation on lattice $\mathcal{L}^{\prime}$}

We want now to know whether percolation occurs in our newly defined discrete model, namely if one can find an infinite open path in $\mathcal{L}^{\prime}$. Let $q$ be the probability that an arbitrary edge is closed. Actually, $q$ is pretty difficult to compute, but we show in next lemma that $q$ can be made arbitrarily small by choosing suitable values of $\lambda$ and $\gamma$.

Lemma 2: For any $q^{\prime}>0$, there exists $\lambda^{\prime}<\infty$ and $\gamma^{\prime}(\lambda)>$ 0 such that

$$
\lambda>\lambda^{\prime} \text { and } \gamma<\gamma^{\prime}(\lambda) \Rightarrow q<q^{\prime} .
$$

Proof: Let us find a lower bound to the probability $p$ that an arbitrary edge of $\mathcal{L}^{\prime}$ is open:

$$
\begin{aligned}
p= & \mathbb{P}\left(\text { an edge of } \mathcal{L}^{\prime} \text { is open }\right) \\
= & \mathbb{P}\left(\left\{2 K^{2} \text { subsquares of surface }(d / K)^{2}\right.\right. \text { have } \\
& \text { at least } 1 \text { point each }\} \text { and }\left\{\text { a surface of } 12 d^{2},\right. \\
& \text { including these subsq, has no more than } N \text { pts }\}) \\
\geq & \mathbb{P}\left(\left\{2 K^{2} \text { subsquares have between } 1\right.\right. \text { and } \\
& \left.\left\lfloor N / 12 K^{2}\right\rfloor \text { pts each }\right\} \text { and }\left\{\text { a surface of } 10 d^{2},\right. \\
& \text { excluding these subsq, has no more than } \\
& \lfloor 5 N / 6\rfloor \text { points }\}) \\
= & \mathbb{P}^{2 K^{2}}\left(\text { a subsq of surface }(d / K)^{2}\right. \text { has between } \\
& \left.1 \text { and }\left\lfloor N / 12 K^{2}\right\rfloor \text { pts }\right) \mathbb{P}\left(\text { a surface of } 10 d^{2}\right. \text { has } \\
& \text { no more than }\lfloor 5 N / 6\rfloor \text { pts }) \\
= & p_{1}^{2 K^{2}}(N) p_{2}(N),
\end{aligned}
$$

where

$$
\begin{aligned}
p_{1}(N)= & \mathbb{P}\left(\text { a subsq of surface }(d / K)^{2}\right. \text { has } \\
& \text { between } \left.1 \text { and }\left\lfloor N / 12 K^{2}\right\rfloor \text { points }\right)
\end{aligned}
$$




$$
\begin{aligned}
= & e^{-\lambda\left(\frac{d}{K}\right)^{2}} \sum_{n=1}^{\left\lfloor N / 12 K^{2}\right\rfloor} \frac{\lambda^{n}}{n !}\left(\frac{d}{K}\right)^{2 n} \\
p_{2}(N)= & \mathbb{P}\left(\text { a surface of } 10 d^{2}\right. \text { has no more than } \\
& \lfloor 5 N / 6\rfloor \text { points }) \\
& e^{-10 \lambda d^{2}} \sum_{n=0}^{\lfloor 5 N / 6\rfloor} \frac{\left(10 d^{2} \lambda\right)^{n}}{n !} .
\end{aligned}
$$

These two quantities admit the following limits:

$$
\begin{aligned}
\lim _{N \rightarrow \infty} p_{1}(N) & =1-e^{-\lambda\left(\frac{d}{K}\right)^{2}} \\
\lim _{N \rightarrow \infty} p_{2}(N) & =1
\end{aligned}
$$

Therefore, if

$$
\lambda>-\left(\frac{K}{d}\right)^{2} \ln \left(1-\left(1-q^{\prime}\right)^{1 / 2 K^{2}}\right):=\lambda^{\prime},
$$

we have

$$
\lim _{N \rightarrow \infty} p(N) \geq\left(1-e^{-\lambda\left(\frac{d}{K}\right)^{2}}\right)^{2 K^{2}}>1-q^{\prime} .
$$

This last inequality means that there exists $N^{\prime}$ such that if $N>N^{\prime}, p>1-q^{\prime}$. On the other hand, we observe in (11) that if $\gamma \rightarrow 0, N \rightarrow \infty$. Hence we can find $\gamma^{\prime}$ such that

$$
\gamma<\gamma^{\prime} \Rightarrow N>N^{\prime} \Rightarrow p>1-q^{\prime} \Rightarrow q<q^{\prime} .
$$

To show the existence of an infinite open path in $\mathcal{L}^{\prime}$, we need the following lemma, which applies to paths in $\mathcal{L}$.

Lemma 3: In $\mathcal{L}$, the probability for a path of length $n$ to be closed is less than or equal to $q^{n / 70}$, where $q$ is the probability that an arbitrary edge is closed.

Proof: Let us consider a path of length $n$ in $\mathcal{L}$ and denote by $S=\left\{\boldsymbol{a}_{i}\right\}_{i=1}^{n}$ the set of the edges forming this path. Let $S^{\prime} \subseteq S$ be a subset of $S$. We clearly have that

$$
\begin{aligned}
\mathbb{P}(\text { the path is closed }) & =\mathbb{P}(\boldsymbol{a} \text { is closed, } \forall \boldsymbol{a} \in S) \\
& \leq \mathbb{P}\left(\boldsymbol{a} \text { is closed, } \forall \boldsymbol{a} \in S^{\prime}\right) .
\end{aligned}
$$

By construction, the event " $a$ is closed" depends on the realization of the Poisson point process in some region of the plane, according to Definition 2. Let us call $R(\boldsymbol{a}) \subset \mathbb{R}^{2}$ this region. It is the rectangle shown in the middle of Figure 8. To compute the probability of this event, we will choose $S^{\prime}$ so that $R(\boldsymbol{a}) \cap R(\boldsymbol{b})=\emptyset, \forall \boldsymbol{a}, \boldsymbol{b} \in S^{\prime}$ and $\boldsymbol{a} \neq \boldsymbol{b}$. In this way, the set of indicator random variables $\mathbb{1}_{\left\{\boldsymbol{a}_{i}\right.}$ is closed $\}$, taking value 1 if with $\boldsymbol{a}_{i}$ is closed and 0 otherwise, with $\boldsymbol{a}_{i} \in S^{\prime}$, are i.i.d variables with $P\left(\mathbb{1}_{\left\{\boldsymbol{a}_{i}\right.}\right.$ is closed $\left.\}=1\right)=q$. Therefore

$\mathbb{P}\left(\boldsymbol{a}\right.$ is closed, $\left.\forall \boldsymbol{a} \in S^{\prime}\right)=q^{m}$, with $m=\operatorname{card}\left(S^{\prime}\right)$.

We construct $S^{\prime}$ as follows: we take the first edge of the path $a_{1} \in S^{\prime}$. This edge is the center of a certain region $R\left(\boldsymbol{a}_{1}\right)$ of the plane, as defined above and shown in Figure 8. Then we follow the path until we find an edge $\boldsymbol{a}_{k}$ such that $R\left(\boldsymbol{a}_{k}\right) \cap R\left(\boldsymbol{a}_{1}\right)=\emptyset$, and add it to $S^{\prime}$. We iterate this last step until we reach the end of the path.

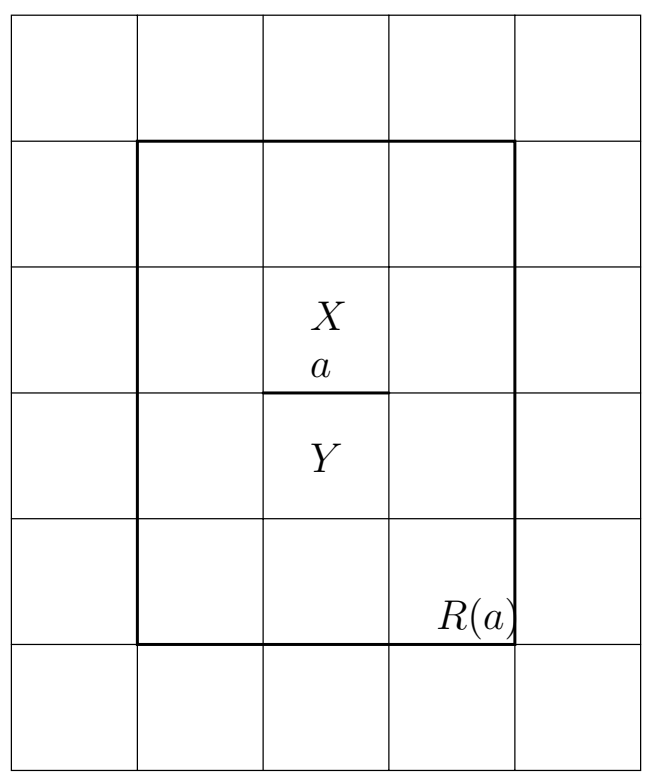

Fig. 8. Edge $a$ and its dependency region $R(a)$. Around $a$, we drew all edges that have a non-disjoint dependency region with $R(a)$.

In order to find an upper bound on $\mathbb{P}\left(\boldsymbol{a}\right.$ is closed, $\left.\forall \boldsymbol{a} \in S^{\prime}\right)$, we need a lower bound on $m=\operatorname{card}\left(S^{\prime}\right)$. In other words, we need to know how many edges are skipped until we find the next element of $S^{\prime}$ in our construction scheme. To answer this question, we will simply count the number of edges $\boldsymbol{b}$ in $\mathcal{L}$ that satisfy $R(\boldsymbol{b}) \cap R\left(\boldsymbol{a}_{1}\right) \neq \emptyset$. We see on Figure 8 that there are 70 of them. We are therefore sure that a path starting with Edge $\boldsymbol{a}_{1}$ cannot go through more than 70 edges without visiting an edge $\boldsymbol{a}_{k}$ such that $R\left(\boldsymbol{a}_{k}\right) \cap R\left(\boldsymbol{a}_{1}\right)=\emptyset$. Since the path $S$ has $n$ edges, $m$ is thus bounded by

$$
m \geq 1+\lfloor(n-1) / 70\rfloor .
$$

We have finally obtained the upper bound we were looking for, and which reads

$$
\begin{aligned}
\mathbb{P}(\text { the path is closed }) & \leq \mathbb{P}\left(\boldsymbol{a} \text { is closed, } \forall \boldsymbol{a} \in S^{\prime}\right) \\
& =q^{m} \\
& \leq q^{1+\lfloor(n-1) / 70\rfloor} \\
& \leq q^{n / 70} .
\end{aligned}
$$

We can now prove the theorem:

Theorem 3: If $q<\left(\frac{11-2 \sqrt{10}}{27}\right)^{70}$, the probability that there exists an infinite open path in $\mathcal{L}^{\prime}$ starting at the origin is strictly greater than zero.

Proof: We will prove this theorem by contradiction: assume that there exists no infinite open path starting at the origin in $\mathcal{L}^{\prime}$. Then there exists a closed circuit in $\mathcal{L}$ that surrounds the origin. In the sequel, we will find an upper bound to the probability that such a circuit exists. The result is then 
deduced from the following equation:

$\mathbb{P}\left(\exists\right.$ an infinite open path starting at the origin in $\left.\mathcal{L}^{\prime}\right)=$ $1-\mathbb{P}(\exists$ a closed circuit in $\mathcal{L}$ that surrounds the origin $)$

We know from [19] (pp. 15-18) that the number $\rho(n)$ of circuits of length $n$ in $\mathcal{L}$ that surround the origin is bounded above by

$$
\rho(n) \leq 4 \cdot n \cdot 3^{n-2}
$$

Among these circuits, some are closed; we denote by $M(n)$ the number of closed circuits of length $n$ in $\mathcal{L}$ that surround the origin. Using Lemma 3, we find a bound to the probability that this number is non zero:

$$
\mathbb{P}(M(n) \geq 1) \leq \rho(n) q^{n / 70} \leq 4 \cdot 3^{n-2} n q^{n / 70}
$$

Consequently,

$$
\begin{aligned}
\mathbb{P}(M(n) \geq 1 \text { for some } n) & =\sum_{n=1}^{\infty} \mathbb{P}(M(n) \geq 1) \\
& \leq \sum_{n=1}^{\infty} 4 \cdot 3^{n-2} n q^{n / 70} \\
& =\frac{4}{3} \hat{q} \sum_{n=1}^{\infty} n\left(3 q^{1 / 70}\right)^{n-1} \\
& =\frac{4 q^{1 / 70}}{3\left(1-3 q^{1 / 70}\right)^{2}} .
\end{aligned}
$$

The above expression is strictly smaller than one if

$$
q<\left(\frac{11-2 \sqrt{10}}{27}\right)^{70}
$$

in which case we can conclude using (13) that:

$\mathbb{P}\left(\exists\right.$ an infinite open path starting at the origin in $\left.\mathcal{L}^{\prime}\right)>0$

\section{Step 3: reverse mapping and percolation on the plane}

Now that there is an infinite open path in $\mathcal{L}^{\prime}$ with positive probability, we still need to prove that this yields the existence of an infinite component in the original graph $\mathcal{G}(\gamma, \lambda)$ for suitable values of $\gamma$ and $\lambda$, as defined in Lemma 2. Lemmas 4 and 5 establish this link between the discrete and the continuous models.

Lemma 4: If a square $X$ is populated, and if the total interference level at any point of the square is less than or equal to $N M$, then all points in the square belong to the same cluster. Furthermore, if two adjacent squares fulfill the same conditions, all points inside these squares belong to the same cluster.

Proof: We consider two adjacent subsquares, and an arbitrary point in each of them (we know that we can find at least one point in each subsquare, because the square is populated). Because both adjacent subsquares have a side of

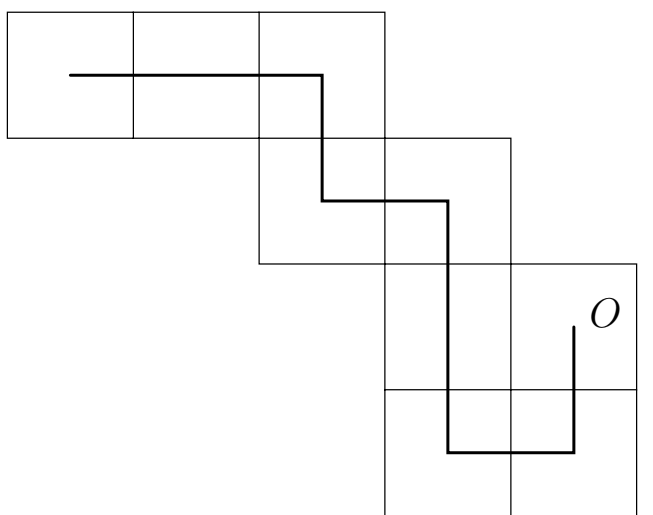

Fig. 9. An open path in $\mathcal{L}^{\prime}$ (in bold) and its associated sequence of squares (whose sides are edges of $\mathcal{L}$ )

length $d / K$, the distance between these two points is at most $\sqrt{5} d / K$. The signal-to-interference ratio is then:

$$
\begin{aligned}
\frac{P_{i} L\left(\boldsymbol{x}_{j}-\boldsymbol{x}_{i}\right)}{N_{0}+\gamma \sum_{k \neq i, j} P_{k} L\left(\boldsymbol{x}_{k}-\boldsymbol{x}_{i}\right)} & \geq \frac{P L\left(\boldsymbol{x}_{j}-\boldsymbol{x}_{i}\right)}{N_{0}+\gamma N M} \\
& \geq \beta
\end{aligned}
$$

where the second inequality follows from (12). Thus each point in a given subsquare is connected to all points in the adjacent subsquares. As a result, since the square is populated, all points in the whole square are connected together.

The second part of the lemma is quite obvious. If two squares are adjacent, we simply consider two adjacent subsquares, one in the first square, one in the other one, and apply the same arguments.

Lemma 5: If there exists an infinite open path in $\mathcal{L}^{\prime}$, then there exists an infinite cluster in the continuous model.

Proof: We consider an infinite open path in $\mathcal{L}^{\prime}$. Remember that each vertex of $\mathcal{L}^{\prime}$ is located at the center of a square of $\mathcal{L}$ (see Figure 9). Along an open path of $\mathcal{L}^{\prime}$, at each vertex, we find a square that fulfills the conditions given in Definition 2. Let us consider one of these squares, which we will denote by $X$. As the attenuation function $L$ is zero for distances above $d$, all interferences in $X$ come from nodes located in $X$ and its direct neighbors (adjacent squares and diagonal neighbors). As the edge is open, according to Definition 2, the total number of points in this neighborhood is less than or equal to $N$. The total interference contribution is thus at most $N M$. We can then apply Lemma 4 and conclude that all points in $X$ are connected together.

Moreover, as two consecutive squares along the infinite open path are adjacent, the sequence of squares form an infinite cluster of connected points.

Combining Lemmas 5, 2 and Theorem 3, we have established Theorem 2 .

\section{A TDMA APPROACH}

We can conclude, from the previous sections, that unless $\gamma$ can be made sufficiently small, long-range communications are impossible if we allow all nodes to emit simultaneously, 
because the graph $\mathcal{G}(\gamma, \lambda)$ may remain in a sub-critical phase for all $\lambda$. Having a small $\gamma$ requires nodes to use CDMA for transmission, which can be complex to implement in an adhoc network (node synchronization may prevent their mobility, etc). An alternative is to avoid having all nodes emitting at the same time, and thus to use a TDMA scheme. We assume that each time interval is divided into $n$ time slots. An optimal TDMA scheme poses also a quite complex challenge to assign the slots to each node, which is clearly beyond the scope of this paper. In this section, we keep the strategy sub-optimal but very simple and totally decentralized: each node picks randomly a number $i$ between 1 and $n$, and only emits during the $i$-th time slot. All nodes are listening at all times. We also assume, for the sake of simplicity, that all nodes emit with the same power $P$.

By applying this TDMA strategy, we reduce the number of interfering nodes by a factor of $n$. It is therefore interesting to compare the connectivity of the graph obtained by superposing the $n$ graphs derived for each slot, to that of the original graph $\mathcal{G}(\gamma / n, \lambda)$ obtained when all nodes emit at the same time with $\gamma n$ times smaller. Let us introduce the following notations for the interference contribution, which is another shot noise, at each time slot:

$$
I_{k}(\boldsymbol{x})=\sum_{i \in S_{k}, \boldsymbol{x}_{i} \neq \boldsymbol{x}} P L\left(\boldsymbol{x}_{i}-\boldsymbol{x}\right)
$$

where $S_{k}, k=1, \ldots, n$ is the set of the indices of the nodes that emit during the $k$-th time slot. It follows immediately that

$$
\sum_{k=1}^{n} I_{k}(\boldsymbol{x})=I(\boldsymbol{x}) .
$$

The expected values of the interference term (1) in the TDMA scheme with $\gamma$ and in the regular scheme with $\gamma / n$ are equal

$$
\mathbb{E}\left[\gamma I_{k}(\boldsymbol{x})\right]=\mathbb{E}\left[\frac{\gamma}{n} I(\boldsymbol{x})\right],
$$

but their variances are different. Indeed, since the points $\boldsymbol{x}_{i}$ are i.i.d. because of the Poisson assumption, we have

$$
\operatorname{VAR}\left[\gamma I_{k}(\boldsymbol{x})\right]=n \operatorname{VAR}\left[\frac{\gamma}{n} I(\boldsymbol{x})\right]
$$

so that the variance of the interference term in the TDMA scheme is $n$ times larger than in the regular scheme. Quite surprisingly, this turns out to improve the connectivity as the percolation threshold $\lambda^{*}(\gamma)$, which is lower with the TDMA scheme. Here is an intuitive explanation for this paradox: at one particular node, consider the value of the interference term (5). Knowing this value, we can compute from (1) the range within which emitting nodes can be heard. This range is large when the interference term has a low value. As the variance of the shot noise is larger in the TDMA case, the probability that it has a very low value is also larger. These nodes for which the interference term is very low are thus able to connect to quite distant nodes, making the emergence of an infinite cluster of connected nodes much more likely in the TDMA case. Of course, many nodes will also have a very high value of the interference term, and will have very few, if any, connections to the rest of the network. But they will not prevent the network to percolate. In the TDMA case, if we superpose the $n$ graphs obtained at each time slot, the resulting graph will have a few long edges essential for its long range connectivity, like in small world graphs [20].

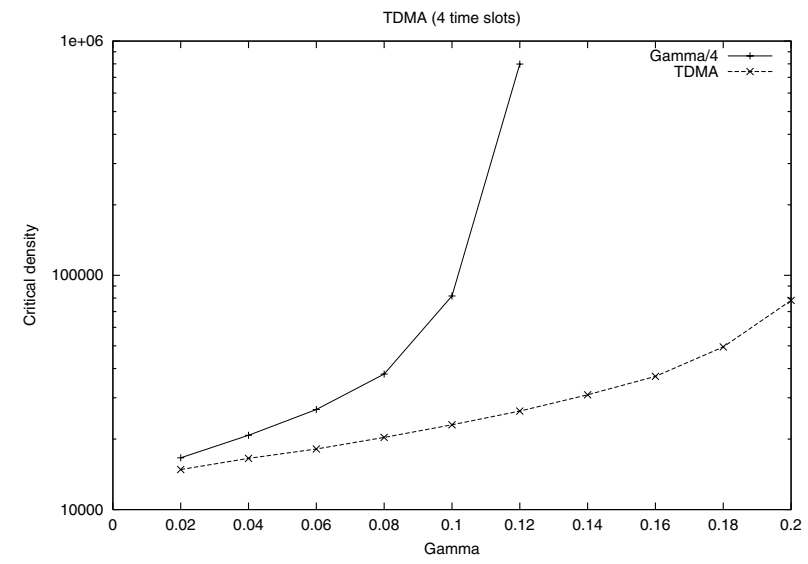

Fig. 10. Comparison between the critical threshold in the TDMA case and in the original model with all nodes allowed to emit simultaneously and with $\gamma$ divided by $n$ [This simulation was run with $n=4, \alpha=3, \beta=1$, $N_{0}=10^{-3}$ and $\left.P_{i}=100,000 \forall i\right]$

Figure 10 shows comparative results for the percolation threshold. We observe indeed that with the TDMA strategy, less nodes are required for percolation than in the original model with all nodes allowed to emit simultaneously and with $\gamma$ divided by $n$. This comparative result shows that dividing $\gamma$ by $n$ in the original model gives a pessimistic approximation of the TDMA strategy. Therefore, it proves that even with pure TDMA, one can move to a supercritical phase (provided that the node density $\lambda$ is large enough). In fact, if we use the strategy described above with $n$ time slots, we know that the connectivity will be better than in our model with $\gamma / n$. Therefore, by increasing $n$, one can move to a case where percolation occurs.

\section{CONCLUSION}

We have studied the connectivity of Poisson Signal To Interference Ratio Graphs (STIRG) $\mathcal{G}(\gamma, \lambda)$ where $\gamma$ represents the imperfect orthogonality of the codes used in CDMA, or is set to 1 in a narrow band system.

The STIRG is radically different from the graph obtained in the Boolean Model, where $\gamma=0$ : the node degree is now bounded (Theorem 1), and the existence of an edge between two nodes depends not only on the location of these two nodes, but on the location of all others. We have shown that if $\gamma$ is too large, all clusters are almost surely finite. Our main result is that percolation, and thus long range communications, are however still possible if $\gamma$ is small enough, but non zero (Theorem 2). If this had not been the case, it would have been a serious impediment for multiple hops large scale ad hoc networks. 
As a small value of $\gamma$ requires very efficient and thus complex CDMA codes, an alternative is to use a TDMA system, where each node emits during 1 slot every $n$ time slots. We found that such a system led to an even better connectivity that the original scheme with an orthogonality factor $\gamma / n$. By making $n$ sufficiently large, we can therefore make the graph reach the super-critical phase using a very simple TDMA scheduling. We gave a qualitative explanation for this quite surprising property; a formal proof is left for future research.

The main restriction in Theorem 2 is the requirement (8) that the attenuation function $L(\cdot)$ has a finite support. This assumption was used in the proof to find an infinite sequence of open independent edges in the lattice $\mathcal{L}^{\prime}$, and to prove bond percolation on this lattice. Our simulations show however that this assumption is not necessary. Future research will address this extension, as well as the shape of the region in the $(\gamma, \lambda)$ plane where percolation occurs.

\section{ACKNOWLEDGMENT}

One anonymous reviewer's comments were very helpful to prepare the final version of this paper.

\section{REFERENCES}

[1] O. Dousse, P. Thiran, and M. Hasler, "Connectivity in ad-hoc and hybrid networks," in Proc. IEEE Infocom, New-York, June 2002.

[2] P. Gupta and P. R. Kumar, "The capacity of wireless networks," IEEE Trans. Inform. Theory, vol. 46(2), pp. 388-404, Mar. 2000.

[3] F. Baccelli and B. Blaszczyszyn, "On a coverage process ranging from the boolean model to the poisson voronoi tessellation, with applications to wireless communications," Adv. Appl. Prob., vol. 33(2), 2001.

[4] Y.-C. Cheng and T. G. Robertazzi, "Critical connectivity phenomena in multihop radio models," IEEE Trans. Commun., vol. 37, pp. 770-777, July 1989.
[5] P. Santi and D. M. Blough, "An evaluation of connectivity in mobile wireless ad hoc networks," in Proc. IEEE DSN, Washington DC, June 2002, pp. 89-98.

[6] C. Bettstetter, "On the minimum node degree and connectivity of a wireless multihop network," in Proc. MobiHoc, Lausanne, Switzerland, June 2002.

[7] F. Xue and P. R. Kumar, "The number of neighbors needed for connectivity of wireless networks," Wireless Networks, submitted for publication.

[8] P. Gupta and P. R. Kumar, "Critical power for asymptotic connectivity in wireless networks," Stochastic Analysis, Control, Optimization and Applications: A Volume in Honor of W.H. Fleming, 1998, edited by W.M. McEneany, G. Yin, and Q. Zhang, (Eds.) Birkhäuser.

[9] E. N. Gilbert, "Random plane networks," SIAM J., vol. 9, pp. 533-543, 1961.

[10] R. Meester and R. Roy, Continuum percolation. Cambridge University Press, 1996.

[11] T. K. Philips, S. S. Panwar, and A. N. Tantawi, "Connectivity properties of a packet radio network model," IEEE Trans. Inform. Theory, vol. 35, pp. 1044-1047, Sept. 1989

[12] S. Quintanilla, S. Torquato, and R. M. Ziff, "Efficient measurement of the percolation threshold for fully penetrable discs," Journal of Physics A, vol. 33(42), pp. L399-L407, Oct. 2000

[13] L. Booth, J. Bruck, M. Franschetti, and R. Meester, "Continuum percolation and the geometry of wireless networks," Annals of Applied Probability, 2002, to be published.

[14] M. Grossglauser and D. Tse, "Mobility increases the capacity of ad-hoc wireless networks," in Proc. IEEE Infocom, Anchorage, Alaska, Apr. 2001.

[15] V. Ramasubramanian, R. Chandra, and D. Mosse, "Providing a bidirectional abstraction for unidirectional adhoc networks," in Proc. IEEE Infocom, Ney-York, June 2002.

[16] V. Veeravalli and A. Sendonaris, "The coverage-capacity tradeoff in cellular cdma systems," IEEE Trans. Veh. Technol., vol. 48, pp. 1443$1451,1999$.

[17] D. J. Daley, "The definition of a multi-dimensional generalization of shot noise," J. Appl. Prob., vol. 8, pp. 128-135, Mar. 1971.

[18] D. Stoyan, W. Kendall, and J. Mecke, Stochastic Geometry and its Applications. Chichester: John Willey \& Sons, 1995.

[19] G. Grimmett, Percolation. Springer, 1999.

[20] D. Watts and S. Strogatz, "Collective dynamics of small-world networks," Nature, vol. 363, pp. 202-204, June 1998. 